\title{
A Review of Vision-Based Vehicle Detection and Tracking Techniques for Intelligent Vehicle
}

\author{
Mengxin $\mathrm{Li}^{1, \mathrm{a}}$, Xiangqian Tian ${ }^{1, \mathrm{~b}}$, Ying Zhang ${ }^{1, \mathrm{c}}, \mathrm{Ke} \mathrm{X}^{1, \mathrm{~d}}$, Dai Zheng ${ }^{1, \mathrm{e}}$ \\ ${ }^{1}$ School of Information and Control Engineering, Shenyang Jianzhu University, Shenyang, 110168, \\ China

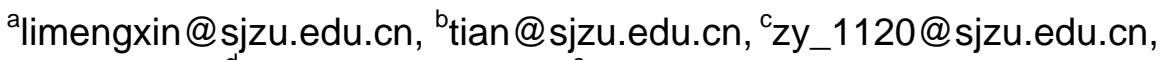 \\ dxuke@sjzu.edu.cn, ${ }^{\mathrm{e}} 374510944 @ q q . c o m$
}

Keywords: Intelligent Vehicle; Machine Vision; Vehicle Detection; Vehicle Tracking

\begin{abstract}
Vision-based vehicle detection and tracking techniques is of great importance to reduce vehicle collision accidents and increase the driving safety on road. This paper presents a comprehensive review of latest techniques for vehicle detection and tracking. In hypothesis generation of vehicles, motion-based, knowledge-based and stereo-vision based methods are introduced. Hypothesis verification includes template-based, appearance-based and multi-features fusion methods. In addition, three main algorithms are introduced in vehicle tracking. Finally, existing problems and future research directions of this field are summarized.
\end{abstract}

\section{Introduction}

As an important part of the intelligent transportation systems and the development direction of future automotive technology, intelligent vehicle have attracted lots of attention of researchers. Many technologies in intelligent vehicle such as object detection and tracking technology, path planning technology and vehicle control technology, are also applied in both military fields and civilian areas.

Vehicle detection and tracking techniques in intelligent vehicle can detect vehicles appearing around the ego vehicle and alert a driver about possible collision with other vehicles, which reduce vehicle collision accidents and increase the driving safety on road. In addition, autonomous navigation based on vehicle detection, vehicle tracking and path planning, eases traffic jams and provides an opportunity for blind people to drive. Computer vision is the most important technique of autonomous navigation system. According to statistics, more than $90 \%$ environmental information is obtained through visual. Visual system provides the most detailed and reliable environmental information for vehicles. From a long-term perspective, vision-based vehicle detection and tracking technologies, will be the future direction of intelligent vehicle navigation technology, and are widely used in all aspects of the intelligent transportation system.

\section{Vehicle Detection for Intelligent Vehicle}

Due to the real time ability requirement, searching possible vehicles in the whole image is impossible. Thus, the detection of vehicles is divided into two main steps: 1) hypothesis generation (HG), which hypothesizes the areas in an image where potential vehicles are; 2) hypothesis verification (HV), which uses certain algorithm to verify the correctness of the hypothesis.

A. Hypothesis generation methods

Three kinds of methods are available to locate potential vehicles:

a. Motion-based method

Motion-based method commonly uses optical flow to estimate the relative motion of vehicles. Optical flow is the instantaneous speed of pixel in the imaging surface. The main idea of the research on optical flow is to calculate the movement of each pixel by using of temporal changes of pixel and their correlation in image sequence. If the optical flow of the ego vehicle is different from other pixels' optical flow, there may be potential moving objects. In [1], a combination of optical 
flow and symmetry tracking was used for vehicle detection. In [2], interest points that persisted over long periods of time were detected as vehicles traveling parallel to the ego vehicle.

b. Knowledge-based method

Knowledge-based method employs a prior knowledge to locate candidate vehicles in an image, such as symmetry, color, texture, corners, vertical/horizontal edges, shadow and parts of the car, such as vehicle lights, windshield and tires. In [3], information about edges was included in the symmetry estimation to filter out homogeneous areas. Literature [4] proposed a vehicle detection system for nighttime, used headlights and taillights for vehicle detection.

c. Stereo-vision based method

Stereovision system can calculate the distance between the detected vehicle and ego vehicle, which also provides understanding of scene, motion characteristics, and physical measurements. There are two types of methods that use the stereo information for vehicle detection. One uses disparity map, while the other uses Inverse Perspective Mapping (IPM). Literature [5] proposed a global optimization algorithm for on-board stereo obstacle detection system. In [6], the dense disparity map was obtained by area-based matching, thus the calculation amount was decreased.

B. Hypothesis verification methods

There are three kinds of methods are widely used in verifying hypothesis:

a. Template-based method

Template-based methods use predefined patterns of the vehicle class and perform correlation between the image and the template. Firstly, this method establishes a template, then searches specified area which matches template mostly, finally achieves the goal of detecting. Bertozzi [7] applied the vertical symmetry of the vehicle to establish templates for matching. Handman [8] used the rectangular with a certain length/width ratio range to verify the hypothesis.

b. Appearance based method

Appearance based $\mathrm{HV}$ is seen as a two-class pattern classification problem: vehicle versus non-vehicle. It contains two steps: 1) Extract some features from the object hypothesis as the classification basis, e. g. PCA (Principal Component Analysis) feature, wavelets feature, Gabor feature, Haar feature and HOG (Histogram of Oriented Gradient) feature. 2) Train the classifier with a series of training data, and input the extracted features to the classifier to identify the object. Commonly used classifiers include neural network, SVM (Support Vector Machine) and Adaboost [9]. SVM and AdaBoost are commonly used in recent years.

c. Multi-features fusion based methods

By fusing different kinds of features, multi-features fusion based methods is adaptable to the environment interference under different conditions. In literature [10], shadows were first used to mark areas that may have a vehicle, edges are then used to eliminate some areas without vehicles and shrink the area. Symmetry was used at last in order to locate the vehicles. Literature [11] fused the edge, texture and vertical edge symmetry on feature-level by weight. The weight values were calculated by separable criterion.

\section{Vehicle Tracking for Intelligent Vehicle}

Vehicle tracking is based on the vehicle detection, according to the vehicle's area of previous frame, using tracking algorithm to confirm the position of the vehicle from the previous frame to the current frame. Three main algorithms are introduced as follow: Mean-shift; Kalman filters; Particle filters.

\section{A. Mean-Shift}

A kind of nonparametric density gradient estimation algorithm based on a general kernel function was proposed, and gave the condition the kernel function shall meet, which can guarantee the estimation is asymptotic unbiased, consistent and continuous with the true value. In [12], Comaniciu applied mean-shift to image analysis. In his work, sample points with zero probability density gradients were found and served as the pattern points of characteristics of spatial clustering model. Literature [13] combined the orientation histogram and HIS model of the object area and used Mean-Shift to search the potential object in the next frame. Literature [14] extracted the 
effective feature by SIFT algorithm, and used the feature point of SIFT as the reference point of Mean-Shift to calculate the center of the object area.

\section{B. Kalman filters}

The Kalman filter, also known as linear quadratic estimation, is an algorithm that uses a series of measurements observed over time, containing noise and other inaccuracies, and produces estimates of unknown variables that tend to be more precise than those based on a single measurement alone. Literature [15] forecasted and tracked the object of the current frame by Kalman filters. Malley [16] detected and tracked the vehicles to the rear by the headlamps, and they used Kalman filter to predict the position of the vehicle in the next frame. Bouttefroy [17] described a new technique for tracking vehicles with mean-shift using a projective Kalman filter.

C. Particle filters

A nonlinear Bayesian filtering method called particle filtering. The basic idea of particle filter is to use a set of random samples with associated weights and estimation based on these samples to represent the posterior probability density. According to Monte Carlo theory, when the number of particle is big enough, the group of particles with associated weight can completely describe a posteriori probability distribution. Takeuchi [18] used particle filters to detect vehicles in complex scenes. Literature [19] proposed a fuzzy-control-based particle filter (FCPF) for maneuvering target tracking, which combines the advantages of standard particle filter and multiple model particle filter. Literature [20] presented a novel compressive particle filter for tracking one or more targets in video using a reduced set of observations.

\section{Existing Problems and Future Prospect}

Although vision-based vehicle detection and tracking techniques for intelligent car has made great progress in recent years, there are some problems and difficulties that are not resolved well. On the one hand, many existing algorithm has its limitations under different weather conditions. On the other hand, when facing multi-vehicles and shelters in images, real time requirement and accurate performance of system is not satisfied. Therefore, the future direction of vehicle detection and tracking techniques can be summarized as follows:

Environment adaptive vehicle detection and tracking system: containing various algorithms to adapt to different environments.

Multi-sensors fused vehicle detection and tracking system: improving the reliability and security by fusing vision sensors with rang sensors which mainly include laser and radar sensors.

\section{Conclusion}

This survey introduced the research progress of vision-based vehicle detection and tracking techniques. HG could be used to get areas in an image where potential vehicles are. HV is to verify the hypothesis using certain algorithms. Vehicle tracking is based on the vehicle detection and estimates the location of vehicle in continuous images. Facing diversity and complexity of real traffic scenes, some methods are hard to satisfy the speed, accuracy and robust demands of system. Algorithm fusion and data fusion provides a good solution to improve the performance of the overall system.

\section{References}

[1] J. Arrospide, L. Salgado, M. Nieto, F. Jaureguizar, On-board robust vehicle detection and tracking using adaptive quality evaluation, 15th IEEE International Conference on Image Processing, p.2008-2011, 2008.

[2] A. Jazayeri, H. Cai, J. Y. Zheng, M. Tuceryan, Vehicle detection and tracking in car video based on motion model, IEEE Transactions on Intelligent Transportation Systems, vol.12, no.2, p.583 -595, Jun.2011. 
[3] M. Bertozzi, A. Broggi, A. Fascioli, Vision-Based intelligent Vehicles: State of the Art and Perspectives, Robotics and Autonomous Systems, vol. 32, p.1-16, Jul.2000.

[4] Y.L. Chen, C.Y. Chiang, Embedded on-road nighttime vehicle detection and tracking system for driver assistance, 2010 IEEE International Conference on Systems, p.1555-1562, 2010.

[5] S. Kubota, T. Nakano, Y. Okamoto, A global optimization algorithm for real-Time on-board stereo obstacle detection systems, proceedings of the 2007 IEEE Intelligent Vehicles Symposium, p. 7-12, 2007.

[6] H. Hattori, N. Takecia, Dense stereo matching in restricted disparity space. 2005 IEEE Intelligent Vehicles Symposium, p.117-122, 2005.

[7] M. Bertozzi, A. Bensrhair, A. Broggi, A cooperative approach to vision-based vehicle detection, IEEE Conference on Intelligent Transportation Systems, p.207-212, 2001.

[8] U. Handmann, T. Kalinke, C. Tzomakas, M. Werner, W. Seelen, An image processing system for driver assistance. Image and Vision Computing, vol.18, no.5, p.367-376, 2000.

[9] Y. Zhu, D. Comaniciu, V. Ramesh, An Integrated Framework of Vision-based Vehicle Detection with Knowledge Fusion, 2005 IEEE Intelligent Vehicles Symposium, vol.2005, p.199-204, 2005.

[10] Y. Zhu, Y. J. Fu, Vehicle detection based on multi-feature fusion. Transducer and Microsystem Technology, vol.28, no.8, p.110-112, 2009.

[11] L.S. Jin, B.Y Gu, R.B. Wang,. Preceding vehicle detection based on multi-characteristics fusion, 2006 IEEE International Conference on Vehicular Electronics and Safety, p.356-360, 2006.

[12] D. Comaniciu, V. Ramesh, P. Meer, Kernel-based object tracking, IEEE Transactions on Pattern Analysis and Machine Intelligence, vol. 25, no. 5, p. 564-577, 2003.

[13] H. Shen, S.M. Li, J.G Mao, A robust vehicle tracking approach using Mean Shift procedure, 5th IEEE International Conference on Information Assurance and Security, p.741-744, 2009.

[14] G. Alessandrettig, A. Broggi, P. Cerri, Vehicle and guard rail detection using radar and vision data fusion, IEEE Transactions on intelligent transportation systems, vol.8, no.1, p.95-105, 2007.

[15] C.Y. Fang, S.W. Chen, C.S. Fuh, Road-sign detection and tracking, IEEE Transactions On Vehicular Technology, p.1329-1341, 2003.

[16] R. O’Malley, M. Glavin, E. Jones, Vision-based detection and tracking of vehicles to the rear with perspective correction in low-light conditions. IET intelligent Transport Systems, vol.5, no.1, p.1-10, 2011.

[17] P.L.M. Bouttefroy, A. Bouzerdoum, S.L. Phung, Vehicle tracking by non-drifting mean-shift using projective Kalman filter, IEEE Conference on Intelligent Transportation Systems, p.61-66, 2008.

[18] A. Takeuchi, S. Mita, D. McAllester, On-road vehicle tracking using deformable object model and particle filter with integrated likelihoods, 2010 IEEE Intelligent Vehicles Symposium, p.1014-1021, 2010.

[19] X.F. Wang, J.F. Chen, Z.G. Shi, K.S. Chen, Fuzzy-control-based particle filter for maneuvering target tracking, Progress in Electromagnetics Research, vol.118, p1-15, 2011.

[20] E. Wang, J. Silva, L. Carin, Compressive Particle Filtering for Target Tracking, 2009 IEEE/SP 15th Workshop on Statistical Signal Processing, p.233-236, 2009. 